\title{
Macrofungi of Küre Mountains National Park in Bartın region of Turkey
}

\author{
Nuri Kaan Özkazançc,"*io, Yağmur Yeşilbaş Keleşa ${ }^{a}$ (i)
}

\begin{abstract}
In this study, Küre Mountains National Park, Bartin Province in the rest of the border must macrofungi flora areas and buffer zones between the years 2013 to 2015 inclusive were searched. The results of the field work space Macrofungal 284 samples were collected. A total of 284 macrofungi samples were collected. Laboratory analysis identified 68 species within 11 orders and 29 families. Of these, 3 taxa belong to Ascomycota, and 65 to Basidiomycota. Determined taxa were found to be growing on four types of substrates: 51 from soil, 11 from branches or trunks, 4 from logs, and 2 from leaves. This study can lay the groundwork for further biodiversity studies in the area, providing a basis for ensuring the continuity of the macromycota flora and biological diversity within the national park.
\end{abstract}

Keywords: Macrofungi, Küre Mountains National Park, Bartın, Mycota

\section{Türkiye’de Küre Dağları Milli Parkının Bartın Bölgesindeki Makrofungusları}

\begin{abstract}
Özet: Bu çalışma ile 2013-2015 yılları arasında Küre Dağları Milli Parkı'nın Bartın İli Sınırlarında kalan bölümünde mutlak ve tampon zon dâhilindeki alanlarda makrofungus türleri araştırılmıştır. Yapılan arazi çalışmaları sonucunda alandan 284 adet makrofungus örneği toplanmıştır. Toplamda sahadan 284 makrofungus örneği toplanmıştır. Labotatuvar ve teşhis çalışmaları sonucunda 10 takım ve 29 familyaya ait toplam 68 tür tanımlanmıștır. Bu türlerden 3 tanesi Ascomycota, 65 tanesi ise Basidiomycota bölümüne dahildir. Toplanan makro funguslardan 51 tanesi topraktan, 11 tanesi dal ve gövdelerden, 4 tanesi kütüklerden ve 2 tanesi de yapraktan olmak üzere 4 farklı substrat üzerinde toplanmıştır. Bu çalışma, bölgede yapılacak olan daha fazla biyolojik çeşitlilik çalışması için zemin hazırlayabilir ve milli parkta makromikota ve biyolojik çeşitliliğin sürekliliğini sağlamak için bir temel oluşturabilir.
\end{abstract}

Anahtar kelimeler: Makrofungus, Küre Dağları Milli Parkı, Bartın, Mikota

\section{Introduction}

Like all the living things in nature, fungi have certain functions and importance within their life-time. For example, $95 \%$ of soil formation is made up of saprophytic fungi (Barutçiyan, 2012). Such as parasitic plants the fungi in a forest, they weed out weaker plants and provide room for the growth of higher quality plants. Some fungi act as nets for various insect species, and mycorrhizal fungi help plants gain mineral matter and water from soil (Palta et al. 2010), and other fungi have been used in medicine and pharmaceuticals (Turp Yildız and Boylu 2018).

Certain fungi, however, can threaten the economy and also be lethal to humans. Eating poisonous fungi is one of the most common health risks (Mat, 1998). Fungi also cause disease in various species, agricultural and forest products, humans and animals alike, which can lead to pecuniary and non-pecuniary losses.

In 1991, a landmark paper estimated that there are 1.5 million fungi on the Earth. Because only 70000 fungi had been described at that time, the estimate has been the impetus to search for previously unknown fungi (Falandysz and Borovicka, 2013). Today, the total number of species is thought to be approximately 5.1 million (Blackwell, 2010). In Turkey, this number is known to be about more than 2500 (Kaya ve Uzun, 2018).
Many mycological studies have been done on macrofungi species in Turkey. Some examples of these studies were presented in Table 1.

Despite the number of studies conducted on macrofungi in Turkey, there is no research on the fungi at Küre Mountains National Park, other than Özkazanç and Yılmaz (2017). Only Afyon et al. (2000), Afyon and Konuk (2002) and Afyon et al. (2005) conducted research on the province of Bartın and its vicinity, but did not focus in particular on Küre Mountains National Park. Özkazanç and Oğuz (2017) identified 45 species in the Kastamonu part of the national park. The selection of Küre Mountains National Park is important for fulling this research gap and contributes to creating a comprehensive inventory of macrofungi distribution in Turkey.

Küre Mountains National Park has a total floor area of 37.753 hectares, $52 \%$ of which (19.500 hectares) are located within the boundaries of Bartın provience. Küre Mountains National Park hosts the best wildlife samples of the endangered "Black Sea Humid Karstic Forest" and is among the 100 forest 'hot spots' in need of protection in Europe. In terms of forest birds, it hosts as many as 129 bird species, the park has also been listed in the "Important Bird Areas" (IBA), and due to the 1050 plant species hosted by the park, it has also been listed as one of the "Important Plant Areas in Turkey" (IPA). Furthermore, Küre Mountains National Park is the only place in the world where 43 plant species live endemically (Görmüș et al. 2015).

\footnotetext{
a Bartın University, Faculty of Forestry, Department of Forest Engineering

@ * Corresponding author (İletişim yazarı): nkaano@gmail.com

$\checkmark \quad$ Received (Geliş tarihi): 24.12.2018, Accepted (Kabul tarihi): 18.03.2019
}

Citation (Atıf): Özkazanç, N.,K., Yeșilbaş Keleş, Y., 2018. Macrofungi of Küre Mountains National Park in Bartın region of Turkey. Turkish Journal of Forestry, 20(1): 8-14. DOI: $10.18182 /$ tjf.501489 
Table 1. Some examples of macrofungi studies in Turkey

\begin{tabular}{|c|c|c|c|c|}
\hline Authors & Year & Site & Total Species count & New species count \\
\hline Sesli & 1993 & Maçka-Trabzon & 40 & 23 \\
\hline Demirel and Işıloğlu & 1993 & Ardanuç-Artvin & 56 & 11 \\
\hline Baydar and Sesli & 1994 & Akçaabat-Trabzon & 40 & 14 \\
\hline Aşkun and Işıloğlu & 1997 & Balya-Balıkesir & 56 & 9 \\
\hline Uzun and Demirel & 1998 & Şenkaya-Erzurum & 50 & - \\
\hline Stojchev et al. & 1998 & Thrace region & 67 & - \\
\hline Demirel and Uzun & 1999 & Sarıkamış-Kars & - & 4 \\
\hline Gezer & 2000 & Antalya & 81 & - \\
\hline Afyon et al. & 2000 & Bartın & 62 & 15 \\
\hline Işıloğlu & 2001 & Sandras Mountain-Muğla & 76 & - \\
\hline Kaya & 2001 & Bitlis & 60 & 4 \\
\hline Demirel et. & 2002 & Ağn1 & 45 & - \\
\hline Afyon and Konuk & 2002 & Zonguldak & 77 & 23 \\
\hline Solak and Yilmaz Ersel & 2002 & Manisa & 36 & - \\
\hline Yabanlı et al. & 2004 & Ula-Muğla & 61 & - \\
\hline Afyon et al. & 2004 & Sinop & 170 & 32 \\
\hline Yilmaz Ersel and Solak & 2004 & Izmir & 55 & 3 \\
\hline Afyon et al. & 2005 & Black Sea region & 80 & 7 \\
\hline Kaya & 2005 & Gölbaşı- Adıyaman & 77 & 5 \\
\hline Doğan and Öztürk & 2006 & Karaman & 202 & - \\
\hline Uzun et al. & 2006 & Gümüşhane & 105 & 14 \\
\hline Aktaş et al. & 2006 & Akseki-Antalya & 85 & - \\
\hline Oskay and Kalyoncu & 2006 & Sultan Mountains & 34 & - \\
\hline Köse et al. & 2006 & Bekilli-Denizli & 61 & - \\
\hline Demir et al. & 2007 & Batman & 50 & 3 \\
\hline Türkoğlu and Gezer & 2007 & Hacer Forest-Kayseri & 69 & - \\
\hline Allı and Ișıloğlu & 2007 & Aydin & 19 & - \\
\hline Yüksel et al. & 2007 & Düzce & 31 & - \\
\hline Kaya et al. & 2009 & Göksun-Kahramanmaraş & 110 & - \\
\hline Türkekul and Zülfikaroğlu & 2010 & Çamlıbel-Tokat & 51 & - \\
\hline Demirel et al. & 2010 & Hatila Valley National Park & 126 & - \\
\hline Küçük et al. & 2010 & Bozyazı-Mersin & 104 & - \\
\hline Kaşık et al. & 2010 & Gevne Valley-Konya & 34 & - \\
\hline Akçay et al. & 2010 & Malazgirt-Muş & 50 & - \\
\hline Alkan et al. & 2010 & Derebucak-Konya & 134 & 6 \\
\hline Gezer et al. & $2011 \mathrm{a}$ & Çamlık-Denizli & 42 & - \\
\hline Gezer et al. & $2011 b$ & Campus of Pamukkale University & 52 & - \\
\hline Kaşık et al. & 2011 & İskilip-Çorum & 54 & - \\
\hline Alli & 2011 & Kemaliye-Erzincan & 106 & 3 \\
\hline Doğan et al. & 2012 & Cocakdere Valley-Mersin & 186 & 4 \\
\hline Solak et. al. & 2013 & Burdur & 35 & - \\
\hline Şen et. al. & 2014 & Bigadiç & 48 & - \\
\hline Keleş et. al. & 2014 & Ayder & 127 & - \\
\hline Güngör et. al. & 2015 & Adan and Mersin & 38 & - \\
\hline Demirel et. al. & 2015 & Van & 122 & - \\
\hline Dengiz and Demirel & 2016 & Şiravn-Siirt & 53 & - \\
\hline Keleş et. al. & 2016 & Kop Mountain & 44 & - \\
\hline Ünal et. al. & 2016 & Mugla & 30 & - \\
\hline Demirel et. al. & 2016 & Lice-Diyarbakır & 55 & - \\
\hline Akata et. al. & 2016 & Zigana Mountain-Gümüşhan & 182 & 6 \\
\hline Demirel ve Koçak & 2016 & Zilan Valley (Erzinca-Van) & 96 & - \\
\hline Akata & 2017 & Belgrat Forest & 280 & - \\
\hline Öztürk et. al. & 2017 & Nallıhan & 68 & - \\
\hline Allı et. al. & 2017 & Yalova & 91 & - \\
\hline Akata and Uzun & 2017 & Uzungöl Nature & 215 & - \\
\hline Altuntaş et. al. & 2017 & Kazdağ1 National Park & 207 & \\
\hline
\end{tabular}

\section{Materials and methods}

In the fieldwork a polyethylene sack for collecting species, knives, gloves, GPS for recording areas, camera, field book for writing the data and sample field cards was used. Stereo and binocular microscopes were used for macro/micro examinations of species which that were collected through field work under proper climate conditions between the years 2013-2015. Photos of the macrofungus species were taken, and their GPS location coordinates were noted, before collected. Geographical properties, morphological structure and ecological properties of the specimens were recorded into the field cards. The specimens were brought into the laboratory and identified via macro/micro examinations. Species identification draw upon previous publications from Petersen 1999, Montag 2000, Seo and Kikr 2000, Williams 2001, Huffman et al. 2008, Lamaison and Polese 2011, and Laux 2012. 


\section{Study sites}

In order to monitor the project and coordinate sample collection, the study field was divided into five main sites and each site as divided into sub-sites based on geographical properties. Table 2 provides GPS data of the five main sites and their sub-sites.

\section{Results}

The identified species are given in Table 3 according to mycobank systematic criteria.

The study identified the following: 68 macrofungus species from two phyla (Ascomycota, Basidiomycota), 11 orders and 29 families. Figure 1 provides species distribution based on their families.

Identified macrofungi were collected from four different substrates: 51 from the soil, 11 from branches or trunks, 4 from $\operatorname{logs}$, and 2 from leaves. The macrofungi were collected from trunks, branches and logs of generally dead or fallen trees, thus imposing no economic loss.

The fourth site (Amasra) yielded the highest number of macrofungus species. Since this area has a large number of broad-leaved forest trees, the moisture and temperature values necessary for the growth and development of macromushrooms are very suitable in this field. Figure 2 provides the number of identified macrofungi species in the research field sites.

Table 2. GPS data of the main and sub-sites of the study field

\begin{tabular}{|c|c|c|c|c|c|}
\hline Site Names & Sub-Sites & Sign & North & East & Altitude \\
\hline \multirow{3}{*}{ 1. Site (Arıt) } & Ören & $1 \mathrm{a}$ & 41॰39'53" & $32^{\circ} 32^{\prime} 25^{\prime \prime}$ & $228 \mathrm{~m}$ \\
\hline & Söğütlü & $1 \mathrm{~b}$ & 41³9'53" & $32^{\circ} 31^{\prime} 21^{\prime \prime}$ & $316 \mathrm{~m}$ \\
\hline & Çöpbey & $1 \mathrm{c}$ & $41^{\circ} 39^{\prime} 32^{\prime \prime}$ & $32^{\circ} 31^{\prime} 23^{\prime \prime}$ & $287 \mathrm{~m}$ \\
\hline \multirow{3}{*}{ 2. Site (Ulus) } & Drahna Valley & $2 \mathrm{a}$ & $41^{\circ} 42^{\prime} 90^{\prime \prime}$ & $32^{\circ} 48^{\prime} 49^{\prime \prime}$ & $519 \mathrm{~m}$ \\
\hline & Kemerli Cave & $2 b$ & $41^{\circ} 42^{\prime} 24^{\prime \prime}$ & $32^{\circ} 49^{\prime} 23^{\prime \prime}$ & $535 \mathrm{~m}$ \\
\hline & Ulukaya & $2 \mathrm{c}$ & $41^{\circ} 40^{\prime} 31^{\prime \prime}$ & $32^{\circ} 46^{\prime} 28^{\prime \prime}$ & $398 \mathrm{~m}$ \\
\hline \multirow{3}{*}{ 3. Site (Sipahiler) } & Sipahiler Cave & $3 a$ & $41^{\circ} 39^{\prime} 32^{\prime \prime}$ & $32^{\circ} 31^{\prime} 23^{\prime \prime}$ & $300 \mathrm{~m}$ \\
\hline & Darı̈̈ren & $3 b$ & 41³9'31" & $32^{\circ} 31^{\prime} 22^{\prime \prime}$ & $295 \mathrm{~m}$ \\
\hline & Çöme Gorge & $3 c$ & 41³9'33" & $32^{\circ} 31^{\prime} 24^{\prime \prime}$ & $264 \mathrm{~m}$ \\
\hline \multirow{3}{*}{ 4. Site (Amasra) } & Karadere Valley & $4 a$ & $41^{\circ} 43^{\prime} 53^{\prime \prime}$ & $32^{\circ} 34^{\prime} 22^{\prime \prime}$ & $487 \mathrm{~m}$ \\
\hline & Yukarışal & $4 \mathrm{~b}$ & $41^{\circ} 43^{\prime} 33^{\prime \prime}$ & $32^{\circ} 34^{\prime} 26^{\prime \prime}$ & $666 \mathrm{~m}$ \\
\hline & Sarıdere & $4 \mathrm{c}$ & $41^{\circ} 43^{\prime} 40^{\prime \prime}$ & $32^{\circ} 34^{\prime} 25^{\prime \prime}$ & $594 \mathrm{~m}$ \\
\hline \multirow{3}{*}{ 5. Site (Kurucaşile) } & Meryemler & $5 a$ & $41^{\circ} 43^{\prime} 56^{\prime \prime}$ & $32^{\circ} 34^{\prime} 21^{\prime \prime}$ & $470 \mathrm{~m}$ \\
\hline & Başköy & $5 b$ & $41^{\circ} 43^{\prime} 58^{\prime \prime}$ & $32^{\circ} 34^{\prime} 24^{\prime \prime}$ & $550 \mathrm{~m}$ \\
\hline & Kömeç & $5 c$ & $41^{\circ} 43^{\prime} 60^{\prime \prime}$ & $32^{\circ} 34^{\prime} 26^{\prime \prime}$ & $651 \mathrm{~m}$ \\
\hline
\end{tabular}

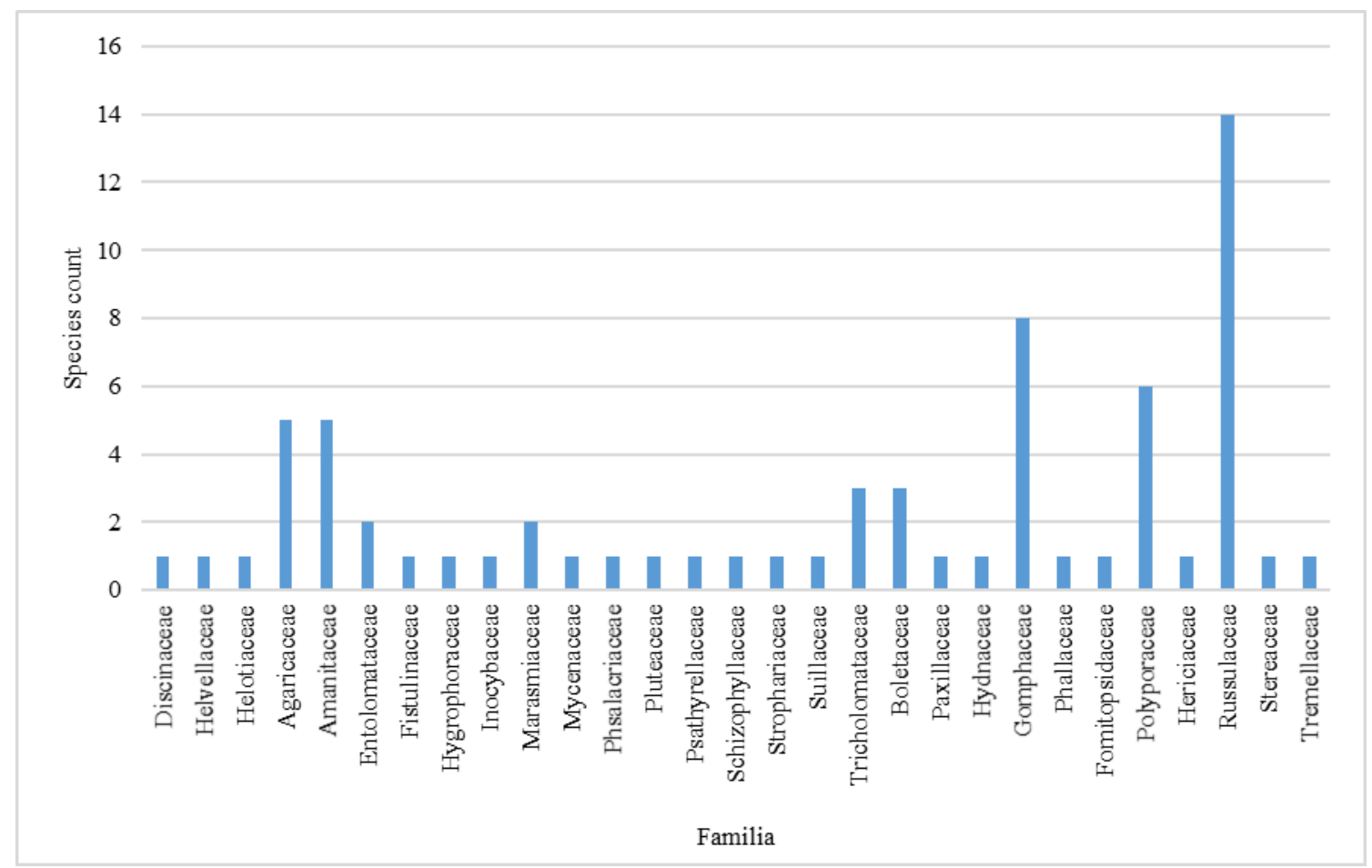

Figure 1. Family-based distribution of the identified species 
Tablo 3. The list of identified macrofungi species of Küre Mountains National Park in Bartın

\begin{tabular}{|c|c|c|c|c|c|c|}
\hline$\cdot \frac{0}{0}$ & Family & Species & Substrate & $\begin{array}{l}\text { Width } \\
(\mathrm{cm})\end{array}$ & $\begin{array}{l}\text { Height } \\
\text { (cm) }\end{array}$ & Site \\
\hline \multirow{3}{*}{ 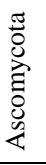 } & Discinaceae & Gyromitra esculenta (Pers.) (Fr.) & soil & $4-10$ & $5-10$ & $4 \mathrm{a}$ \\
\hline & Helvellaceae & Helvella crispa Bull. & soil & $3-7$ & $6-15$ & $2 b$ \\
\hline & Helotiaceae & $\begin{array}{l}\text { Hymenoscyphus calyculus (Sowerby) W. } \\
\text { Phil. }\end{array}$ & trunk & 2 & 4 & $3 b$ \\
\hline \multirow{20}{*}{ 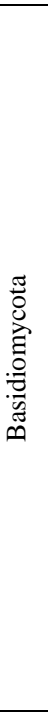 } & \multirow{5}{*}{ Agaricaceae } & Bovista aestivalis (Bonord.) Demoulin & soil & 4 & 4 & $1 b-4 c$ \\
\hline & & Bovista plumbea Pers. & soil & $1-3$ & $1-3$ & $1 \mathrm{~b}$ \\
\hline & & Coprinus comatus (O.F. Müll.) Pers. & soil & $6-15$ & 4-7 & $2 \mathrm{c}$ \\
\hline & & Lycoperdon perlatum Pers. & soil & $4-5$ & $4-5$ & $1 b-1 c-4 a$ \\
\hline & & Lycoperdon pyriforme Willd. & soil & $4-5$ & $4-5$ & $1 \mathrm{~b}$ \\
\hline & \multirow{5}{*}{ Amanitaceae } & Amanita muscaria (L.) Lam. & soil & $10-20$ & $10-20$ & $2 \mathrm{c}$ \\
\hline & & Amanita phalloides (Fr.) Link. & soil & $6-12$ & $5-8$ & $4 \mathrm{~b}$ \\
\hline & & Amanita pantherina (DC.) Krombh. & soil & $6-10$ & $6-10$ & $1 \mathrm{~b}$ \\
\hline & & Amanita rubescens Pers. & soil & $10-20$ & $10-20$ & $1 \mathrm{~b}$ \\
\hline & & Amanita velosa (Peck) Lloyd & soil & $5-11$ & $4-11$ & $4 \mathrm{~b}$ \\
\hline & \multirow{2}{*}{ Entolomataceae } & Entoloma griseocyaneum (Fr.) P. Kumm. & soil & $2-5$ & $4-7$ & $3 \mathrm{a}$ \\
\hline & & Entoloma sericellum (Fr.) P. Kumm & soil & $1-2$ & $2-6$ & $3 b$ \\
\hline & Fistulinaceae & Fistulina hepatica (Schaeff.) With. & trunk & $10-12$ & $3-5$ & $4 \mathrm{~b}$ \\
\hline & Hygrophoraceae & Hygrophorus agathosmus (Fr.) Fr. & soil & $5-7$ & $3-7$ & $4 \mathrm{c}$ \\
\hline & Inocybaceae & Inocybe asterospora Quel & soil & 7 & 5 & $4 \mathrm{a}$ \\
\hline & \multirow{2}{*}{ Marasmiaceae } & Marasmius androsaceus (L.) Fr. & soil & $0,5-1$ & $5-6$ & $4 \mathrm{c}$ \\
\hline & & Marasmius oreades (Bolton) Fr. & soil & $3-7$ & $6-7$ & $3 a$ \\
\hline & Mycenaceae & Mycena crocata (Schrad.) P. Kumm. & on litter & $1-2$ & $10-12$ & $4 \mathrm{a}$ \\
\hline & Physalacriaceae & Armillaria mellea (Vahl) P. Kumm. & $\begin{array}{l}\text { bottom of trunk and on } \\
\text { soil }\end{array}$ & $4-7 \mathrm{~cm}$ & $10-15$ & $1 c-4 c$ \\
\hline & Pluteaceae & Pluteus cervinus (Schaeff.) P. Kumm. & on soil & $5-10$ & $2-7$ & $4 \mathrm{a}$ \\
\hline \multirow{29}{*}{ 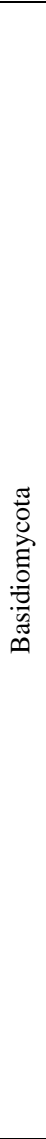 } & Psathyrellaceae & $\begin{array}{l}\text { Coprinellus disseminatus (Pers.) } \\
\text { Lange }\end{array}$ & on dead wood & $1-2$ & $2-4$ & $3 \mathrm{a}$ \\
\hline & Schizophyllaceae & Schizophyllum commune Fr. & on dead wood block & 4 & $0,3-0,5$ & $1 a-1 b-2 c$ \\
\hline & Strophariaceae & Pholiota squarrosa (Oeder) P. Kumm & on wood & $4-10$ & $1-2$ & $2 \mathrm{c}$ \\
\hline & \multirow{3}{*}{ Tricholomataceae } & Collybia cirrhata (Schumach.) Quel. & dead foliage & $1-5$ & & $3 \mathrm{c}$ \\
\hline & & Collybia conigena (Pers.) P. Kumm. & $\begin{array}{l}\text { on litter and dead } \\
\text { foliage }\end{array}$ & $1-3$ & $2-4$ & $4 \mathrm{~b}$ \\
\hline & & $\begin{array}{l}\text { Collybia ocior (Pers.) Vilgalys \& O.K. } \\
\text { Mill. }\end{array}$ & $\begin{array}{l}\text { on litter and dead } \\
\text { foliage }\end{array}$ & $1,5-5$ & & $2 \mathrm{c}$ \\
\hline & Boletaceae & Boletus erythropus Pers. & soil & $10-13$ & $12-18$ & $3 a-4 b$ \\
\hline & & Boletus luridus Schaeff. & soil & $6-15$ & $5-14$ & $4 \mathrm{c}$ \\
\hline & & Boletus smithii Thiers & soil & $10-16$ & $5-17$ & $4 \mathrm{c}$ \\
\hline & Paxillaceae & Paxillus rubicundulus P.D. Orton & soil & 10 & & $4 \mathrm{c}$ \\
\hline & Suillaceae & Suillus luteus (L.) Roussel & soil & $8-10$ & $5-8$ & $3 a$ \\
\hline & Hydnaceae & Hydnum repandum $\mathrm{L}$. & soil & $8-20$ & $5-15$ & $4 \mathrm{c}$ \\
\hline & \multirow{8}{*}{ Gomphaceae } & Clavariadelphus occidentalis Methven & soil & $3-5$ & $5-20$ & $4 \mathrm{c}$ \\
\hline & & Clavariadelphus pistillaris L. (Donk) & soil & $2-6$ & $8-20$ & $4 \mathrm{c}$ \\
\hline & & $\begin{array}{l}\text { Clavariadelphus sachalinensis (S. Imai) } \\
\text { Corner }\end{array}$ & soil & & 7 & $4 \mathrm{c}$ \\
\hline & & Clavariadelphus truncatus (Quel.) Donk. & soil & & $10-13$ & $4 \mathrm{c}$ \\
\hline & & Ramaria aurea (Schaeff.) Quel & soil & & $7-14$ & $4 \mathrm{~b}$ \\
\hline & & Ramaria flava (Schaeff.) Quel & soil & $7-15$ & $10-20$ & $4 \mathrm{a}$ \\
\hline & & Ramaria formosa (Pers.) Quel & soil & & $8-20$ & $4 \mathrm{a}$ \\
\hline & & Ramaria stricta (Pers.) Quel & soil & & $5-10$ & $2 \mathrm{c}$ \\
\hline & Phallaceae & Clathrus ruber P. Micheli ex Pers. & soil & $3-5$ & $10-15$ & $3 a$ \\
\hline & Fomitopsidaceae & Daedalea quercina (L.) Pers. & trunk & $3-12$ & $4-5$ & $1 \mathrm{a}$ \\
\hline & \multirow{6}{*}{ Polyporaceae } & Lentinus strigosus Fr. & wood & $2-10$ & $1-4$ & $2 \mathrm{~b}$ \\
\hline & & Lenzites betulina (L.) Fr. & trunk & 10 & $1-2$ & $2 \mathrm{c}$ \\
\hline & & Polyporus mori (Pollini) Fr. & trunk & $2-6$ & $2-6$ & $2 \mathrm{a}$ \\
\hline & & Trametes hirsuta (Wulfen) Pilat & trunk & 10 & 6 & $2 \mathrm{a}$ \\
\hline & & $\begin{array}{l}\text { Trametes ochracea (Pers.) Gilb. \& } \\
\text { Ryvarden }\end{array}$ & trunk & $1,5-5$ & & $4 \mathrm{a}$ \\
\hline & & Trametes versicolor (L.) Lloyd & trunk & $2-8$ & $2-8$ & $2 c-4 b$ \\
\hline & Hericiaceae & Creolophus cirrhatus (Pers.) P. Karst. & trunk & $6-8$ & $5-13$ & $2 \mathrm{a}$ \\
\hline
\end{tabular}


Table 3. Continunes The list of identified macrofungi species of Küre Mountains National Park in Bartın

\begin{tabular}{|c|c|c|c|c|c|c|}
\hline$\cdot \frac{\Xi}{0}$ & Family & Species & Substrate & $\begin{array}{l}\text { Width } \\
(\mathrm{cm})\end{array}$ & $\begin{array}{l}\text { Height } \\
(\mathrm{cm})\end{array}$ & Site \\
\hline \multirow{16}{*}{ 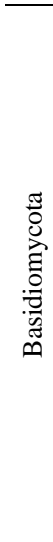 } & \multirow{14}{*}{ Russulaceae } & Lactarius barrowsii Hesler \& A.H. Sm. & soil & $5-10$ & & $5 b$ \\
\hline & & Lactarius controversus Pers. & soil & $10-20$ & $8-18$ & $3 a$ \\
\hline & & Lactarius deliciosus (L.) Gray & soil & $10-15$ & $3-6$ & $1 \mathrm{~b}$ \\
\hline & & Lactarius sanguifluus (Paulet) Fr. & soil & $10-15$ & $5-7$ & $4 \mathrm{a}$ \\
\hline & & Lactarius vellereus (Fr.) Fr. & soil & $10-25$ & $10-20$ & $1 \mathrm{~b}$ \\
\hline & & Russula aquosa Leclair, Bull. & soil & $5-15$ & $3-7$ & $3 a$ \\
\hline & & Russula cyanoxantha (Schaeff.) Fr. & soil & $5-15$ & $6-10$ & $4 \mathrm{c}$ \\
\hline & & Russula delica Fr. (1838) & soil & $2-20$ & $2-5$ & $1 \mathrm{~b}$ \\
\hline & & Russula drimeia Cooke & soil & $5-12$ & $4-10$ & $4 \mathrm{a}$ \\
\hline & & Russula emetica (Schaeff.) Pers.; & soil & $5-10$ & $4-8$ & $4 \mathrm{a}$ \\
\hline & & Russula fageticola (Romagn.) Bon & soil & $6-10$ & $4-10$ & $4 \mathrm{a}$ \\
\hline & & Russula lepida Fr. & soil & $10-12$ & $5-10$ & $1 a-1 b-1 c$ \\
\hline & & Russula sanguinea (Bull.) Fr. & soil & $4-10$ & $4-10$ & $4 a$ \\
\hline & & Russula turci Bres. & soil & $4-8$ & $4-10$ & $1 \mathrm{~b}$ \\
\hline & Stereaceae & Stereum hirsutum (Willd.) Pers. & trunk & & $2-5$ & $4 \mathrm{~b}$ \\
\hline & Tremellaceae & Tremella mesenterica (Schaeff.) Retz. & dead; trunk & $3-12$ & $3-12$ & $2 \mathrm{a}$ \\
\hline
\end{tabular}

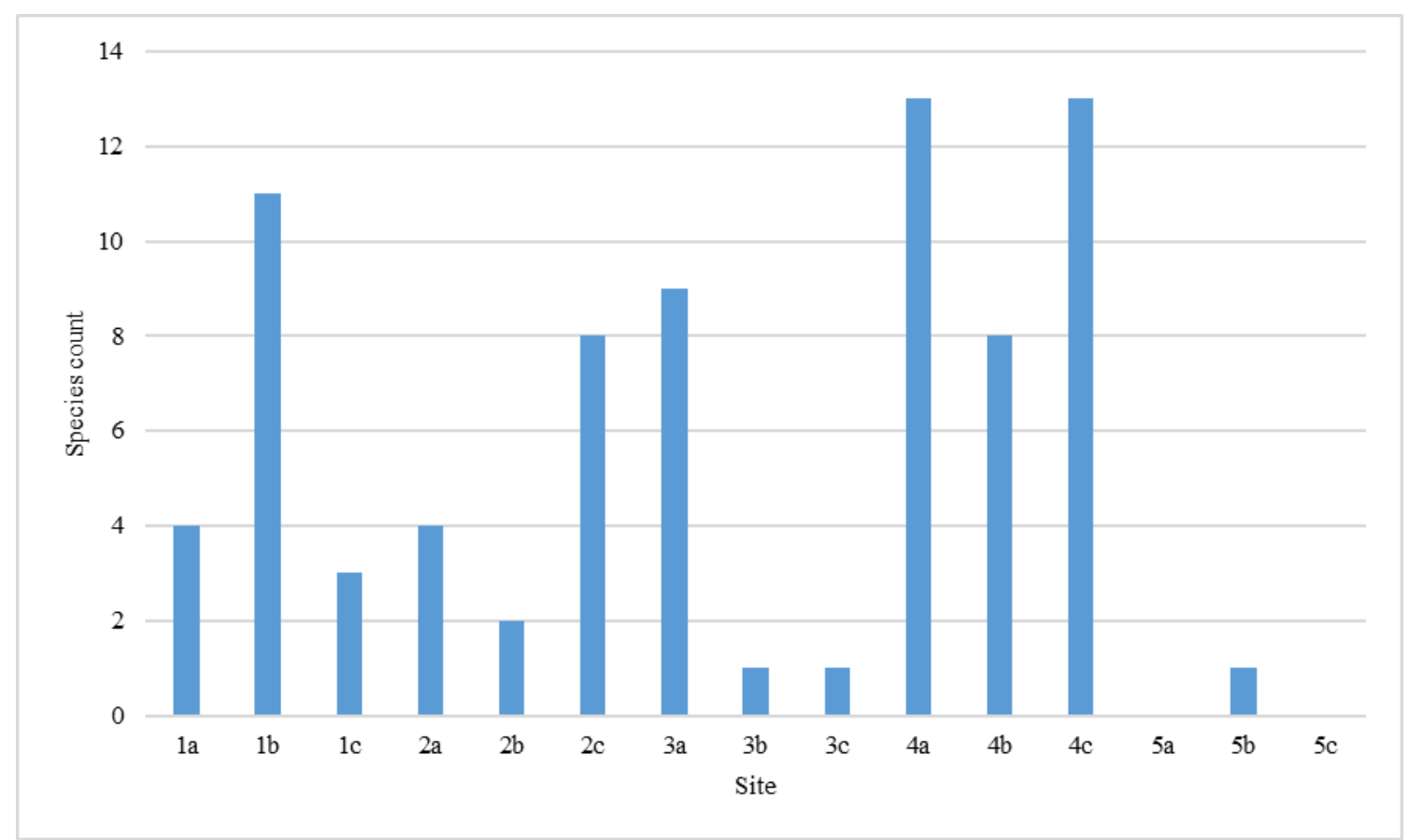

Figure 2. Ditribution of the determined taxa to the assigned sites

\section{Discussion}

Afyon et al. (2000) identified 62 macrofungus taxa belonging to 19 families in a study they conducted in the province of Bartın, 15 of which were taxa newly recorded for the macrofungi of Turkey. In other research, Afyon et al. (2005) identified macrofungi of the western Black Sea region, including two sites from the Bartın region, which yielded two different species from their previous research. In our research, 69 macrofungus taxa were identified solely in Küre Mountains National Park and recorded for the province of Bartın. Afyon et al. (2000) identified only one species belonging to the class Ascomycetes. Our research increases this number to four. Only six of the species identified in the study that Afyon et al. (2000) conducted (Suillus luteus, Trametes hirsuta, Ramaria aurea, Lactarius controversus, L. deliciosus, Schizophyllum comune) were also identified in our research. In comparison, the remaining 62 taxa were new recordings for the province of Bartın.

In light of the aforementioned information, it seems possible that new studies, whether in the province of Bartın or Küre Mountains National Park, can lead to an increase in the identified number of species and new recordings for the macrofungus flora of Turkey.

\section{Acknowledgment}

This study was supported by the Coordinatorship of Scientific Research Projects of Bartın University as project no. BAP 2012108. We would like to express our thanks to the Bartın University Rectorship and the Coordinatorship of Scientific Research Projects for their much-appreciated support. 


\section{References}

Afyon, A., Konuk, M., 2002. Zonguldak yöresi makrofungusları üzerine bir araştırma. Ot Sistematik Botanik Dergisi, 9(1):121128.

Afyon, A., Konuk, M., Yağı,, D., 2000. Bartın yöresi makrofungusları üzerinde bir araştırma. Selçuk Üniversitesi Eğitim Fakültesi. Fen Bilimleri Dergisi, 8(2): 77-86.

Afyon, A., Yağız, D., Konuk, M., 2004. Macrofungi of Sinop province. Turkish Journal of Botany 28: 351-360.

Afyon, A., Konuk, M., Yağız, D., Stephan, H., 2005. A study of wood decaying macrofungi of the western Black Sea Region Turkey. Mycotaxon, 93:319-322.

Akata, I., 2017. Macrofungal diversity of Belgrad Forest (İstanbul). Kastamonu Uni., Orman Fakültesi Dergisi, 17 (1): 150-164.

Akata, I., Uzun, Y., 2017. Macrofungi determined in Uzungöl Nature Park (Trabzon). Trakya University Journal of Natural Sciences, 18(1): 15-24, DOI: 10.23902/trkjnat.295542.

Akata, I., Uzun, Y., Kaya, A., 2016. Macrofungal diversity of Zigana Mountain (Gümüşhane/Turkey). Biological Diversity and Conservation, 9(2): 57-69.

Akçay, E., Uzun, Y., Kaya, A., 2010. Malazgirt (Muş) yöresi makrofunguslarına katkılar. Mantar Dergisi (The Journal of Fungus), 1(1):14-20.

Aktaş, S., Öztürk, C., Cevizci, M., 2008. Akseki (Antalya) ilçesi makrofungusları. 19. Ulusal Biyoloji Kongresi, 23-27 June 2008, Trabzon, p. 450.

Alkan, S., Kaşık, G., Aktaş, S., 2010. Derebucak (Konya-Türkiye) ilçesi makrofungusları, TÜBİTAK Turk J Bot., 34: 335-350.

All, H., 2011. Macrofungi of Kemaliye district (Erzincan). Turkish Journal of Botany, 35: 299-308, DOI:10.3906/bot-1004-298.

Allı, H., Işıloğlu, M., 2007. Türkiye makrofunguslarına Aydın yöresinden yeni kayıtlar. ÇEV-KOR, 16(64): 63- 73.

All, H., Candar, S. S., Akata, I., 2017. Macrofungal diversity of Yalova Province. Mantar Dergisi- The Jounal of Fungus, 8(2): 76-84.

Altuntaş, D., Allı, H., Akata, I., 2017. Macrofungi of Kazdağ1 National Park (Turkey) and its close environs. Biological Diversity and Conservation, 10(2): 17-25.

Aşkun, T., Işıloğlu, M., 1997. Macrofungi of Balya (Balıkesir) county. Turkish Journal of Botany, 21: 279-284.

Barutçiyan, J., 2012. Türkiye'nin Mantarları. 1. Baskı, Oğlak Güzel Kitaplar, 216 s. ISBN: 9789753297677, İstanbul.

Baydar, S., Sesli, E., 1994. Trabzon ili Akçaabat yöresinde belirlenen makromantarlar. Turkish Journal of Botany, 18: 99101.

Blackwell, M., 2010. The Fungi. American Journal of Botany, 98(3): 426-438.

Demir, S., Demirel, K., Uzun, Y., 2007. Batman yöresi makrofungusları. ÇEV-KOR, 16 (34), 37- 42.

Demirel, K., Acar, İ., Boztepe Ömeroğlu, G., 2016. Lice (Diyarbakır) yöres makrofungusları. Mantar Dergisi- The Jounal of Fungus, 7(1):29-39 DOI $: 10.15318$ /Fungus.2016118352.

Demirel, K., Erdem, Ö., Uzun, Y., Kaya, A., 2010. Macrofungi of Hatila Valley National Park (Artvin, Turkey). Tübitak Turk J. Bot., 34:457-465, DOI:10.3906/Bot-0908-189.

Demirel, K., Isıloglu, M., 1993. Ardanuç (Artvin) yöresi makrofungusları (I). Yüzüncü Y1l Üniversitesi Fen Bilimleri Dergisi, 4 (4): 49-57.

Demirel, K., Koçak, Z., 2016. Zilan Vadisi'nin (Erciş-VAN) makrofungal çeşitliliği. Mantar Dergisi- The Jounal of Fungus, 7(2): 122-134, DOI :10.15318/Fungus.2016222680.

Demirel, K., Uzun, Y., 1999. Sarıkamış (Kars) yöresinden Türkiye mantar florası için yeni kayıtlar. Ot Sistematik Botanik Dergisi, 6, 1:83-88.

Demirel, K., Uzun, Y., Akçay, M. E., Keleş, A., Acar, İ., Efe, V., 2015. Van yöresi makromantarlarına katkılar. Mantar Dergisi (The Jounal of Fungus), 6(2):13-23 DOI: 10.15318/Fungus.2015214060.
Demirel, K., Uzun, Y., Kaya, A., 2002. Ağr1 bölgesinin makrofungusları. Yüzüncü Yıl Üniversitesi, Biyoloji Bölümü, Tübitak Turk J. Bot., 26:291-295.

Denğiz, Y., Demirel, K., 2016. Şirvan (Siirt) yöresinde yetişen makrofunguslar üzerinde taksonomik bir araştırma. Yüzüncü Y11 Üniversitesi Fen Bilimleri Enstitüsü Dergisi (Journal of The Institute of Natural \& Applied Sciences), 21 (2): 112-123.

Doğan, H. H., Aktaş, S., Öztürk, C., Kaşık, G., 2012. Macrofungi distribution of Cocakdere valley (Arslanköy, Mersin). Turkish Journal of Botany, 36: 83-94, DOI:10.3906/bot-1010-9.

Doğan, H. H., Öztürk, C., 2006. Macrofungi and Their Distribution in Karaman Province, Turkey Turkish Journal of Botany, 30: 193-207.

Falandysz, J., Borovicka, L. 2013. Macro and trace mineral constituents and radionuclides in mushrooms: health benefits and risks. Appl Microbiol Biotechnol., 97(2): 477-501.

Gezer, K., 2000. Contributions to the macrofungi flora of Antalya province. Turkish Journal of Botany, 24: 293-298.

Gezer, K., Kaygusuz, O., Soylu, U., Ermiş, A., 2011a. Çamlık Mesire Alanı (Denizli) makrofungusları. Pamukkale Üniversitesi, Fen Edebiyat Fakültesi Biyoloji Bölümü, Mantar Dergisi (The Jounal of Fungus), 2(1-2): 15-24.

Gezer, K., Kaygusuz, O., Soylu, U., Ermiş, A., 2011b. Macrofungi of Pamukkale University Kınıklı Campus (Denizli/Turkey). Biological Diversity and Conservation, 4/3: 36-431.

Görmüş, S., Atmış, E., Artar, M., Özkazanç, N. K., Günşen, H. B., Cengiz, S., Tekebaş, S., 2015. Küre Dağları Milli Parkı Köy Tasarım Rehberleri (Bartın Bölümü) Bartın Üniversitesi Yayınları No: 20 Orman Fakültesi Yayınları No: 10 x,234 sayfa ISBN: 978-605-9895-05-7 Mutlu Basım Yayın, Bartın.

Güngör, H., Solak, M. J., Allı, H., Işılloğlu, M., Kalmış, E., 2015. Adana ve Mersin yöresi makrofungus çeşitliliğine katkılar. Mantar Dergisi (The Jounal of Fungus), 6(2):38-42 DOI: 10.15318/Fungus.2015214063.

Huffman, D. M., Tiffany, H. L., Knaphus, G., Healy, R. A., 2008. Mushrooms and Other Fungi of the Midcontinental United States Second Edition. University of Iowa Press. ISBN 978-158729-627-7.

Iş1loğlu, M., 2001. Sandras Dağı (Muğla) makrofungusları. Selçuk Üniversitesi Eğitim Fakültesi, Fen Bilimleri Dergisi, 9: 127136.

Kaşık, G., Aktaş, S., Öztürk, C., Doğa, H. H., 2010. Macrofungi distrubition of Gevne Valley. Mantar Dergisi (The Jounal of Fungus), 1-2: 25-32.

Kaşık, G., Uçar, S., Aktaş, S., 2011. İskilip (Çorum) ilçesi makrofunguslar1. Mantar Dergisi (The Jounal of Fungus),1-2: 9-13.

Kaya, A., 2001. Contributions to the macrofungi flora of Bitlis Province. Turkish Journal of Botany, 25: 379-383.

Kaya, A., 2005. Macrofungi determined in Gölbaşı (Adıyaman) District. Turkish Journal of Botany, 29: 45-50.

Kaya, A., Uzun, Y., 2018. New contributions to the Ascomycota. Turkish Journal of Botany, 42: 644-652.

Kaya, A., Uzun, Y., Karacan, H. İ., 2009. Macrofungi of Göksun (Kahramanmaraş) District. Turkish Journal of Botany, 33: 131139.

Keleş, A., Demirel, K., Uzun, Y., Kaya, A., 2014. Macrofungi of Ayder (Rize/Turkey) high plateau. Biological Diversity and Conservation, 7(3): 177-183.

Keleş, A., Polat, T., Demirel, K., 2016. Kop Dağı'ndaki bazı yenilebilir mantarlar (Erzurum-Bayburt). Türk Tarım - Gıda Bilim ve Teknoloji Dergisi, 4(3): 221-224.

Köse, S., Gezer, K., Gökler, I., Türkoğlu, A., 2006. Macrofungi of Bekilli (Denizli) district. Turkish Journal of Botany, 30: 267272.

Küçük, M. A., Doğan, H. H., Akata, I., 2010. A Study on macrofungal diversity of Bozyazı province (Mersin), Turkey. Gazi University Journal of Science, 23(4):393-400.

Lamaison, J., Polese, J., 2011. Atles il-lustrat dels Bolets, Susaeta Press. ISBN: 978-84-677-0035-0, 240s. 
Laux, H. E., 2012. Setas De Espana Y Europa. 718 p. Tikal Ediciones Pres ISBN:978-84-9928-168-1 Madrid.

Mat, A., 1998. Türkiye'de Mantar Zehirlenmeleri Zehirli Mantarlar. 1. basım, TÜBİTAK yayınları, TÜBİTAK Matbaas1, Ankara.

Montag, K., 2000. Pilze, Kosmos Kompakt, ISBN: 3-440-07835-3 Franckh-Kosmos Verlags-GmbH \& Co. KG Stuttgard.

Oskay, M., Kalyoncu, F., 2006. Contribution to the macrofungi flora of Sultan Mountain, Turkey. International Journal of Science \& Technology, 1(1): 7-10.

Özkazanç, N. K., Oğuz Yılmaz, M., 2017. Küre Dağları Milli Parkı'nın Kastamonu ili sınırlarında kalan bölümünün Makrofungusları. Kastamonu Univ., Orman Fakültesi Dergisi, 17 (4): 643-651 Doi: 10.17475/kastorman.369219.

Öztürk, C., Pamukçu, D., Aktaş, S., 2017. Nallıhan (Ankara) İlçesi Makrofungusları Mantar Dergisi (The Jounal of Fungus), 8(1): 60-67 Do :10.15318/Fungus.2017127497.

Palta, Ş., Demir, S., Şengönül, K., Kara, Ö., Şensoy, H., 2010. Arbüsküler mikorizal funguslar (amf), bitki ve toprakla ilişkileri. Bartın Orman Fakültesi Dergisi, 12(18): 87-98.

Petersen, J. H., 1999. Key to the genera of clavarioid fungi in Northern Europe, https://www.mycokey.com/MycokeyDK/ DKkeysPDFs/ClavarioidGenusKey.pdf.

Şen, İ., Allı, H., Işılloğlu M., 2014. Bigadiç (Balıkesir) yöresi makrofunguslar1. Mantar Dergisi, 5(2): 9-16 Doi :10.15318/Fungus.201428230.

Seo, G. S., Kirk, P. M., 2000. Ganodermacea, nomenclature and classification. Ganodermacea Diseaese of Perennial Crops. ISBN 9780851993881.

Sesli, E., 1993. Trabzon İli Maçka yöresi makrofungusları. Turkish Journal of Botany, 17(3): 179-182.

Solak, H. M., Yilmaz Ersel F., 2002. Manisa yöresinden makrofungus florasına kayıtlar. Ekoloji Çevre Dergisi, 10 (43): $30-32$
Solak, M. H., Allı, H., Ișıloğlu, M., Güngör, H., Kalmış, E., 2013. Macrofungi of Burdur province Mantar Dergisi (The Jounal of Fungus), 4(2): 28-34.

Stojchev, G., Aslan, A., Gücin, F., 1998. Some macrofungi species of European part of Turkey. Turkish Journal of Botany, 22: 341-346.

Turp Y1ldı, G., Boylu, M., 2018. T1bbi ve yenilebilir mantarlar\& et ürünlerinde kullanımı. YYÜ Tar. Bil. Derg., 28(1):144-153.

Türkekul, İ., Zülfikaroğlu, E., 2010. Çamlıbel ilçesi (Tokat) makromantar floras. Sakarya Üniversitesi Fen Edebiyat Dergisi, 2010-I: 55-63.

Türkoğlu, A., Gezer, K., 2007. Hacer Ormanı (Kayseri)'nin makrofungusları. Pamukkale Üniversitesi, Eğitim Fakültesi, Fen Bilgisi Bölümü, Çev-kor dergisi, Ekoloji, 15(59): 43-48.

Ünal, G., Türkoğlu, A., Güngör, M., 2016 Muğla yöresindeki eucalyptus ormanlarında yetişen makrofunguslar üzerine taksonomik çalıșmalar. Türk Tarım - Gıda Bilim ve Teknoloji Dergisi, 4(3): 244-247.

Uzun, Y., Demirel, K., 1998. Şenkaya (Erzurum) ilçesinin makrofungusları. XIII. Ulusal Biyoloji Kongresi, Bildiriler Kitab1, Cilt 1, 7-10 Eylül 1998, Samsun, s.213-222.

Uzun, Y., Keleş, A., Demirel, K., 2006. Contributions to the macrofungi flora of Gümüşlhane province. Turkish Journal of Botany, 30: 39-46.

Williams, J., 2001. Simplified Fung1 Identiification Key. Universty of Georiya Cooperative Extension Service Special Bulletin, 37.

Yabanlı, M., Işıloğlu, M., Solak, M. H., 2004. Ula (Muğla) yöresinde tespit edilen bazı yenen makrofunguslar. XVII. Ulusal Biyoloji Kongresi, 21-24 Haziran, Adana. s. 78.

Yilmaz Ersel, F., Solak, M. H., 2004, Contributions to the macrofungi of İzmir province. Turkish Journal of Botany, (28) 487-490.

Yüksel, B., Akbulut, S., Baysal, İ., Gültekin, Y. S., 2007. Düzce yöresinin yenilebilir mantarları. I. Uluslarası Odun D1şı Orman Ürünleri Sempozyumu, 1-4 Kasım 2016 Trabzon, Volume: 1. 